Original Research Paper

\title{
Use of Lazy Wavelet and DCT for Vibration Signal Compression
}

\author{
1*Aimé Joseph Oyobe Okassa, ${ }^{1}$ Jean Pierre Ngantcha, ${ }^{1}$ Auguste Ndtoungou and ${ }^{2}$ Pierre ELE \\ ${ }^{1}$ Department of Mechanical Engineering, EPM, Université des Sciences et Techniques de Masuku, Gabon \\ ${ }^{2}$ Department of Telecommunications, ENSP of UYdé, University of Cameroon, Cameroon
}

\author{
Article history \\ Received: 13-11-2020 \\ Revised: 05-01-2021 \\ Accepted: 08-01-201 \\ Corresponding Author: \\ Aimé Joseph Oyobe Okassa \\ Department of Mechanical \\ Engineering, EPM, Université \\ des Sciences et Techniques de \\ Masuku, Gabon \\ Email: oyobeokassa@yahoo.fr
}

\begin{abstract}
In this study, we compress and decompress the vibration signals from the functioning of a ball bearing. The methodology used to compress the vibration data in this study is to reduce the size of the data by reducing the spectral redundancy of the samples. We have used the DCT, which is recognized for its representational parsimony and bleaching power. To reduce the execution time of the algorithm, we used the Lazy wavelet. This wavelet separates the original signal into two signals half the size of the original signal. Parallel processing of two halves of the original signal reduces the computational load of the algorithm. We tested (compressed and then decompressed) these signals using three compression algorithms separately under the same quantification and coding conditions. These are the algorithms based on DCT, WHT and the Lazy Wavelet associated with DCT. The comparison made on the basis of the measurements of SNR, MFD, MSE, PRD and CR allowed to retain the algorithm based on the use of the Lazy wavelet and the discrete cosine transform. The results are considered very encouraging.
\end{abstract}

Keywords: Lazy Wavelet, DCT, Compression, Vibration Signals, Storage, Bandwidth, Rotating Machines

\section{Introduction}

Industrial maintenance is a sector that is developing more and more with the improvement of the machines. Unexpected outages are sometimes very expensive. The loss of production during repairs creates a shortfall that can affect the company's profits. It becomes clear that such failures should not be tolerated. Vibration analysis consists of detecting possible malfunctions and monitoring their evolution in order to plan or to defer a mechanical intervention. However, experience has shown that the most reliable parameter that gives the earliest and best manner the state of deterioration of a rotating machine is vibration. Indeed, the vibratory signal is the support of the indicators (physical quantities) used regularly to monitor the installations. The evolution of vibrations makes it possible to alert the technician to a degradation of the functioning of an industrial installation. For this, we distinguish scalar or global level indicators (NG) and shape or spectral indicators. All machines vibrate and as the condition of the machine deteriorates (clumsy, bearing or bearing defect....) the vibration level increases. By measuring and monitoring the level of vibration produced by a machine, an ideal indicator of its condition is obtained. If the increased vibration of the machine makes it possible to detect a defect, the analysis of the vibration characteristics of the machine makes it possible to identify the cause (Chaib et al., 2004).

The objective of our work is to compress the vibration signals that come from the operation of a ball bearing. This compression allows to optimize the storage space of these data and to improve their transmission speed. To this goal, several compression techniques are proposed. We can mention the scalar/vector quantification, differential coding, predictive coding and transformed coding (Salleh and Soraghan, 2007; Jolivet and Stouls, 1972; Pesquet and Tziritas, 1988; Trabuco et al., 2017). Among these techniques, those using transforms offer better results (Iqbal et al., 2007). The use of transform coding is justified by the fact that points adjacent to a signal can be strongly correlated. The efficiency of a decorrelation depends on the type of transformation used (destructive compression). In terms of compression all non-detectable elements by human sensors are removed (Non-destructive compression) (Aimé et al., 2019). Thus, (Sunitha and Chitneedi, 2014) compressed the voice using the Discrete Wavelet Transform (DWT) supplemented by Kalman's adaptive filtering. Their aim was to improve audibility in mobile communication. Shnain et al. (2020) 
to improve the compression ratio of the EZW algorithm, they used their modified version of EZW for lossless compression. Abo-Zahhad et al. (2015) presented a new approach of medical images compression. This approach is based on the pre-processing of images using the DPCM method. The result of this pretreatment is decomposed by DWT. Wavelet coefficients are then encoded by Huffmann. Chaoang et al. (2020) presented a new reconstruction method for vibration signals from the bearings of an axial piston pump. This algorithm is based on the sparse adaptive dictionary model. Narayan et al. (2019) explored the potential relationship between various combustion events monitored using a pressure transducer in the cylinder and vibration using an accelerometer. The resulting data was compressed. Despite the abundance of work in a very current field such as signal compression, an algorithm composed of a Lazy Wavelet (LW) and a DCT has not yet been tested on vibratory signals.

The originality of this study lies in the improvement of both the compression ratio while maintaining the quality of the reconstructed signal and the compression time. We cannot forget the comparison under the same conditions of quantization and coding of algorithms based on DCT, WHT and the LW associated with DCT. This comparison specifies the compression capabilities of each of these algorithms.

This article consists of three parts: Materials and Methods, Results and Discussion.

\section{Materials and Methods}

\section{Generalities on the Compression}

Compression of the data allows the reduction of the data size while preserving a better quality of the reconstructed data. There are two types of compression: Lossless compression that allows a perfect reconstruction of information but for very low compression ratios and lossy compression that improves compression ratio at the expense of reconstructed information quality (Huffman, 1952; Fernandes and Jeberson, 2014). The general principle of compression/decompression is shown in Fig. 1.

Figure 1 shows the general data compression/decompression procedure. Depending on the type of compression envisaged, the components of the encoder change. In the case of lossless compression, the original signal is simply encoded to reduce its size. If the compression is lossy, the coder necessarily contains a mathematical application. This application, often orthogonal, projects the data in a space with a better representation (parsimony). This change of space allows the removal of non-detectable information by human sensors and redundant ones (bleaching). In both cases, the decompression follows the same algorithm but in the opposite direction.

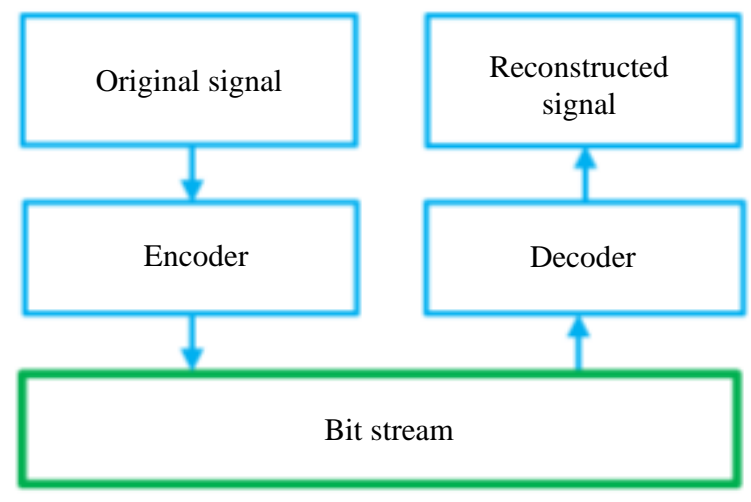

Fig. 1: Compression/decompression chain

\section{Compression Evaluation Parameters}

The evaluation of a compression algorithm is done through two groups of parameters: Quantitative and qualitative parameters. The quantitative parameter often used is the compression ratio. Its value is defined by Eq. (1):

$R C=\left(1-\left(\frac{\text { size of compressed file }}{\text { size of original file }}\right)\right)$

Compression ratio defines the ability of a compression algorithm to reduce data size.

The quality of the reconstructed information is given by Eqs. ( 2 to 5$)$ :

- Mean Quadratic Error (MSE) is the parameter that expresses the qualitative difference between the original and reconstructed signal. It is defined by Eq. (2):

$$
M S E=\frac{1}{N} \sum_{n=1}^{N}\left(s_{0}(n)-s_{r}(n)\right)^{2}
$$

$s_{0}(n)=$ The original signal

$s_{r}(n)=$ The reconstructed signal

$N=$ The number of samples of the signal

- The Signal-to-Noise Ratio (SNR) is the parameter that expresses the energy conservation of the signal and defines by:

$$
S N R=10 \log \left(\frac{\sigma_{x}^{2}}{\sigma_{e}^{2}}\right)
$$

with, $\sigma_{x}^{2}$ representing the power of the original signal and $\sigma_{e}^{2}$ representing the power of the error: 
- Mean Frequency Distorsion (MFD)

$$
M F D=\left(\frac{\left|F_{\text {orig }}-F_{\text {recons }}\right|}{\max \left(F_{\text {orig }}, F_{\text {recons }}\right)}\right)^{2}
$$

In the expression (4), $F_{\text {orig }}$ and $F_{\text {recoms }}$ represent the average frequency calculated respectively on the original signal and on the reconstructed signal:

- The PRD (Percent Root mean square Difference):

$$
P R D=\sqrt{\frac{\sum_{n=0}^{N-1}\left(s_{0}(n)-s_{r}(n)\right)^{2}}{\sum_{n=0}^{N-1}\left(s_{0}(n)-\mu\right)^{2}}} .100 \%
$$

$N=$ The number of points of the original signal

$\mu=$ The Analog-to-Digital Converter (ADC) reference value used for the acquisition of $s(n)$ and $\mu=0$ data

\section{Proposed Method}

\section{Compression/Decompression Procedure}

The compression/decompression scheme implemented in our algorithm is given in Fig. 2.
Figure 2 shows the proposed compression algorithm. In this algorithm, the signal is separated into two signals by the lazy wavelet. This separation is done by taking into account the sample index in the original signal. All odd indices form a signal and even indices form a second signal. Each signal is decomposed by the DCT. The coefficients obtained are quantized. Quantization limits the number of bits to be transferred. The Huffman encoding allows transforming the values of the coefficients obtained into a binary train (coded data). Reconstruction of the signal follows the Fig. 3.

Figure 3 shows the data decompression scheme according to our algorithm. Here, we have to go through the same steps as those realized in the case of compression but in reverse.

\section{Discrete Wavelets Transform}

In mathematics, a wavelet $\Psi$ is a square-integrable functions from the space of Hilbert $L^{2}(\mathbb{R})$, most often oscillating and zero-averaging, chosen as a multi-scale analysis and reconstruction tool. We define a family $\psi_{s, \tau}$ where $(s, \tau) \in \mathbb{R}^{+*} x \mathbb{R}$ of wavelets from the mother wavelet $\Psi$ :

$\forall t \in \mathbb{R}, \psi_{s, \tau}(t)=\frac{1}{\sqrt{s}} \Psi\left(\frac{t-\tau}{s}\right)$

where, $s$ is scaling factor and $\tau$ represents time shift factor.

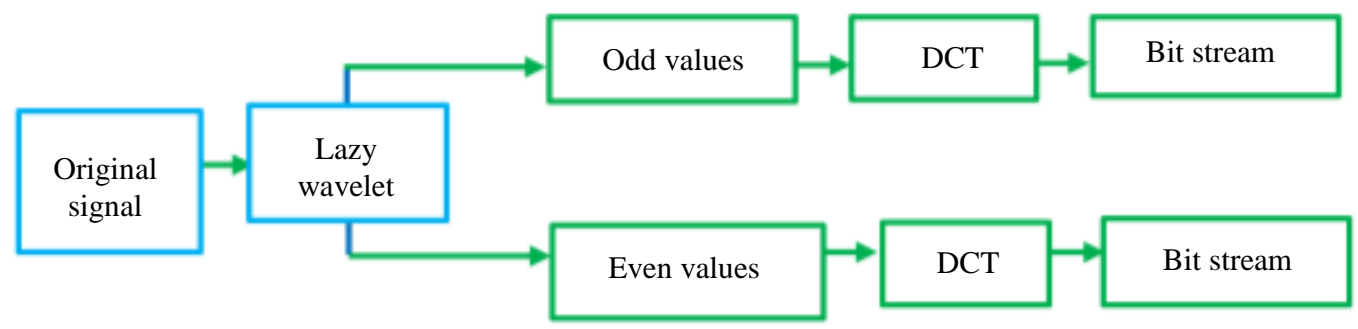

Fig. 2: Proposed compression algorithm

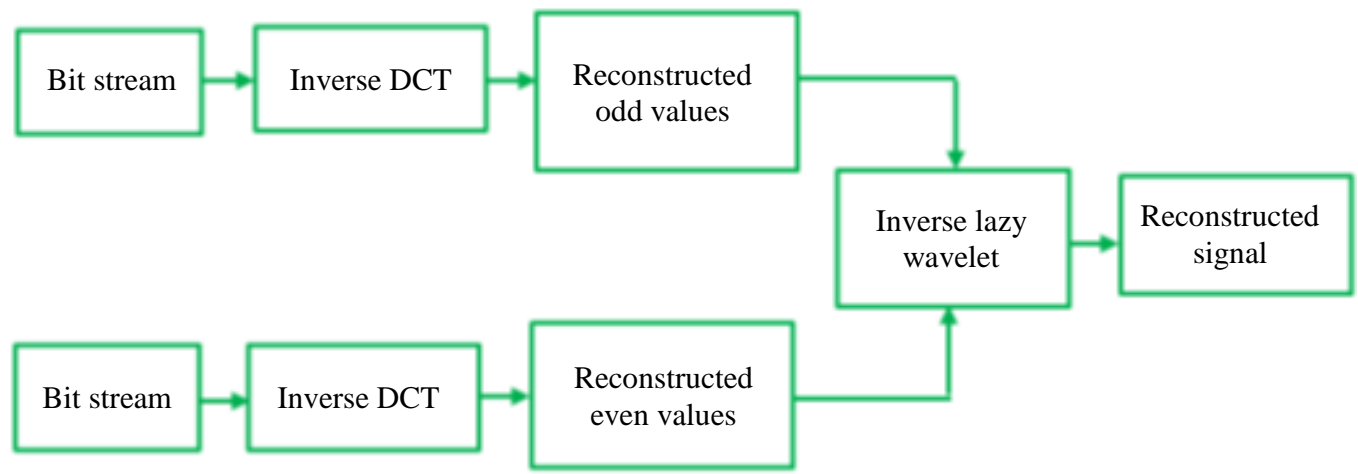

Fig. 3: Proposed algorithm of decompression 
To analyze a square-integrable function in wavelets consists in calculating all its scalar products with the wavelets family. The resulting numbers are called wavelet coefficients and the operation associating its wavelet coefficients to a function is called wavelet transform. The continuous wavelet transform of a function $f \in L^{2}(\mathbb{R})$ is defined by:

$$
g(s, \tau)=\int_{-\infty}^{+\infty} f(t) \psi_{s, \tau}^{*}(t) d t
$$

For compression applications the Discrete Wavelet Transform (DWT) is used. This transformation is defined by the following equations:

$$
\begin{aligned}
& a[j-1, k]=\sum_{k=1}^{n} h[n-2 k] a[j, n] \\
& d[j-1, k]=\sum_{k=1}^{n} g[n-2 k] a[j, n] \\
& H[\omega]=\sum_{k=1}^{n} h[k] e^{-j k \omega} \\
& G[\omega]=\sum_{k=1}^{n} g[k] e^{-j k \omega}
\end{aligned}
$$

In the Eqs. (8 to 11) $G[\omega], H[\omega], a[j-1, k], d[j-1, k]$ are respectively the low pass filter, the high pass filter, the approximation coefficients and the detail coefficients.

The reconstruction of the signals is done by Eq. (12):

$$
a[j, k]=\sum_{n}^{N} h[n-2 k] a[j-1, k]+\sum_{n}^{N} g[n-2 k] a[j-1, k]
$$

\section{Discrete Cosine Transform (DCT)}

The DCT is a transformation close to the Discrete Fourier Transform (DFT). The projection nucleus is a cosine and gives real coefficients, unlike the DFT, whose nucleus is a complex exponential and which gives complex coefficients. The most common variant of the Discrete Cosine Transform is the DCT Type-II, often simply referred to as the "DCT". Its inverse, which corresponds to type-III is often simply called "IDCT".

A discrete cosine transformation expresses a finite sequence of data points in terms of a sum of cosine oscillating functions at different frequencies. These direct and inverse transformations are defined respectively by Eqs. (13) and (14) (Wheidima et al., 2016):

$$
\begin{aligned}
& y(k)=\alpha(k) \sum_{n=0}^{N-1} x(n) \cos \left[\frac{\pi}{N}\left(n+\frac{1}{2}\right) k\right] \\
& k=0,1,2, \ldots . N-1 \\
& x(n)=\sum_{n=0}^{N-1} \alpha(k) y(k) \cos \left(\frac{(2 n+1) k \pi}{2 N}\right) \\
& k=0,1,2, \ldots . N-1
\end{aligned}
$$

where, $x(n)$ is sample $n$.

The advantage of this transformation is that this transformation has a fast calculation algorithm and allows to obtain real coefficients.

The method presented in this article has been implemented on a real vibratory signal. These are records of the vibration monitoring of a ball bearing. The reference for this ball bearing is SKF7309B. The acquisition system consists of a portable collector, VIBROTEST 60 and an accelerometer. The signals were acquired with a sampling frequency of $2 \mathrm{kHz}$. The acquisition device model uses a 12-bit Analog-to-Digital Converter for scanning acquired data. The vibration parameter chosen for this work is the amplitude variation.

To ensure compressibility of these data, we can calculate the autocorrelation coefficient. This coefficient is defined by the relation (16):

$$
R(k)=\sum_{n}\left(x_{n}-m\right)\left(x_{n-k}^{*}-m\right)
$$

In this formula $x_{n}, x_{n-k}^{*}$ and $m$ are respectively sample $n$, sample $n$ shifted by $k$ samples and the average of samples calculate on the original signal. The $k$-order autocorrelation coefficient is simply the Pearson coefficient calculated between a series and itself offset by $k$ elements. It represents the cross-correlation of a signal by itself. The autocorrelation makes it possible to detect regularities, repeated profiles in a signal. The value of the correlation coefficient is between -1 and +1 . For the data processed in this study, the value of this coefficient is equal to 0.2529 . This value indicates that the studied signal is weakly correlated. In these conditions, simple coding is therefore not appropriate for the compression of this data. So to compress this signal we will use the space change. DCT has better ability to bleach and compact data. The lazy wavelet allows a separation of tasks. This task separation reduces the number of data to be manipulated for a task. Each task processes half of the original data.

\section{Results}

After compressing/decompressing these signals using the lazy wavelet followed by DCT, we obtained the results which are compared with DCT and WHT algorithms and are recorded in Table 1 . The results of the compression/decompression of the vibration data by the algorithm that we propose are presented in Fig. 2. 


\section{Discussion}

The results in Table 1 present the qualitative and quantitative differences between the reconstructed signals by the methods object of comparison and those of the proposed method. The variation amplitudes of the used signals vary between 1 and 2 micrometers. The Mean Square Errors (MSE) are for the three methods in the nanometric order and therefore negligible. Moreover, this precision is difficult to achieve in mechanical engineering. The algorithm based on the DCT and the proposed method better conserve the signal energy respectively 42.88 and 43.32. The PRD of the three methods is equal. This equality is linked to the fact that the MSE is very small and of the Nanometric order. The mean frequency distortion of the three methods is very near to zero (0). The results presented in Fig. 4 are those of the proposed method. The capabilities of this method are appreciated by the compression parameters whose values are: $\mathrm{SNR}=43.32 \mathrm{~dB}, \mathrm{MSE}=1.66 \mathrm{E}-05, \mathrm{MFD}$ $=0.1 \%$ and $\mathrm{CR}=95.43 \%$. The reconstruction error of the proposed method is a constant value and almost equal zero. This confirms from an objective point of view the good quality of the reconstructed data. The method offers a high compression ratio. Much of this improvement is achieved by reducing the spectral redundancy of samples using DCT. Indeed, the DCT has good representational parsimony and a great power of data bleaching. Using the Lazy wavelet separates the original signal into two signals half the size of the original signal. Parallel processing of two halves of the original signal reduces the computational load of the algorithm. This is another advantage of this algorithm which combines the Lazy wavelet and the DCT.

Table 1: Comparison of algorithms

\begin{tabular}{llllll}
\hline Algorithme & MSE $(\mu m)$ & SNR $(\mathrm{dB})$ & PRD $(\%)$ & MFD $(\%)$ & CR $(\%)$ \\
\hline DCT & $9.2 \mathrm{E}-06$ & 42.88 & 2.18 & 0.00 & 43.32 \\
WHT & $23 \mathrm{E}-04$ & 18.99 & 2.18 & 0.05 & 71.94 \\
Proposed method & $1.66 \mathrm{E}-05$ & 43.32 & 2.18 & 0.10 & 95.43 \\
\hline
\end{tabular}
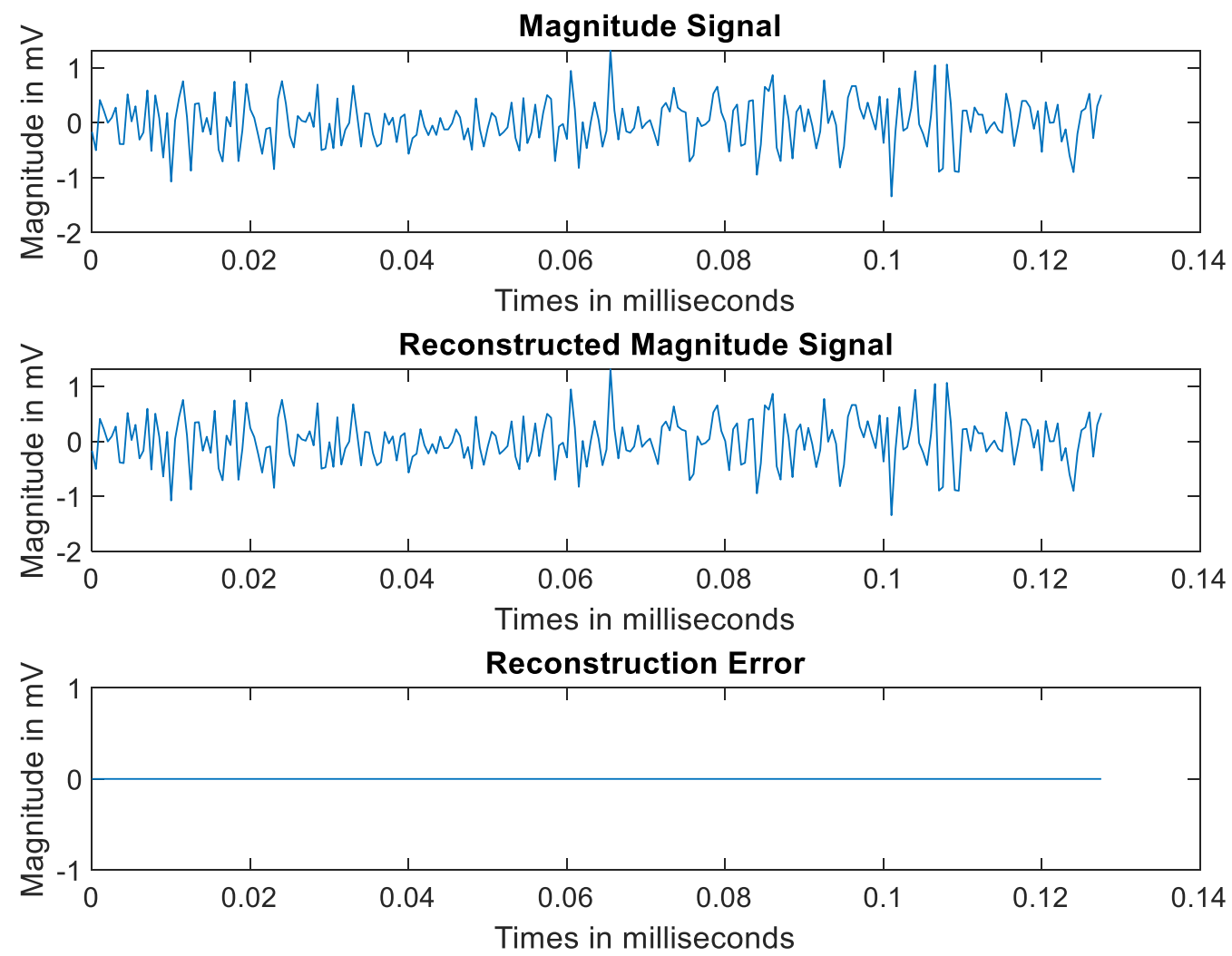

Fig. 4: Compared representation of signal: Original, reconstructed and error 


\section{Conclusion}

In this article, we presented a method for compressing/decompressing vibration data. From the Table 1, the proposed method gives very good results both qualitatively and quantitatively. The near-zero mean square error is a particularity of this algorithm based on the compression by transform methods. Despite the absence of refined quantization (1.E-02), this error is null. The size of the quantification step limits the number of bits to be transmitted, thus influencing the computational complexity of the algorithm. Thus, we can say that our algorithm is of low computational complexity (for a sample of 256 points). The results obtained by this algorithm are encouraging with regard to the objective and subjective criteria (SNR, MSE, MFD, CR and visual observation). The compression ratios of this algorithm are high (95.43\%). However, reference should also be made to the opinion of the mechanical engineer who specializes in rotating machinery maintenance. Optimizing storage space and improving the transmission speeds of vibration data can be a means of improving the results of vibration analysis in industrial maintenance.

\section{Author's Contributions}

Aimé Joseph Oyobe Okassa: Worked with algorithm implementation, text writing and participated in all experiments, coordinated the-analysis.

Jean Pierre Ngantcha: Worked with algorithm implements, in all experiments and contributed to the writing of the manuscript.

Auguste Ndtoungou: Participated in all experiments and coordinated the data-analysis and reviewing.

Pierre ELE: Contributed to the methodological aspects and revised the final version of the manuscript.

\section{Ethics}

I affirm that the manuscript has been prepared in accordance with Science Publication's Journals instructions to authors and the content of this manuscript, or a major portion thereof, has not been published in a referred journal nor being submitted for publication elsewhere.

\section{References}

Aimé, J. O. O., Joseph, M. N., \& Pierre, E. 2019. Compression of the EMG signals by WalshHadamard Transform associated with the Predictive Coding DPCM. International Journal of System Control and Engineering Application, 12(1), 1-7

Abo-Zahhad, M., Gharieb, R. R., Ahmed, S. M., \& AbdEllah, M. K. (2015). Huffman image compression incorporating DPCM and DWT. Journal of Signal and Information Processing, 6(02), 123.
Chaib, R., Meziani, S., \& Verzea, I. (2004). Surveillance des roulements par analyse vibratoire. Sciences \& Technologie. B, Sciences de l'ingénieur, 23-27.

Chaoang, X., Hesheng, T., \& Yan, R. (2020). Compressed sensing reconstruction for axial piston pump bearing vibration signals based on adaptive sparse dictionary model. Measurement and Control, 53(3-4), 649-661.

Fernandes, A., \& Jeberson, W. (2014, March). An image compression technique using wavelet. In Proceedings of the IEEE Tech Sym Satellite Conference organized by VIT-Vellore in association with IIT Kharagpur.

Huffman, D. A. (1952). A method for the construction of minimum-redundancy codes. Proceedings of the IRE, 40(9), 1098-1101.

Iqbal, M. A., Javed, M. Y., \& Qayyum, U. (2007, November). Curvelet-based image compression with SPIHT. In 2007 International Conference on Convergence Information Technology (ICCIT 2007) (pp. 961-965). IEEE.

Jolivet, J. C., \& Stouls, F. X. (1972, May). Codage différentiel d'images multiniveaux, application au visiophone. In Annales des Télécommunications (Vol. 27, No. 5-6, pp. 225-236). Springer-Verlag.

Narayan, S., Milojevic, S., \& Gupta, V. (2019). Combustion monitoring in engines using accelerometer signals. Journal of Vibroengineering, 21(6), 1552-1563.

Pesquet, J. C., \& Tziritas, G. (1988, October). Adaptive Methods Applied To Intra And Interframe Predictive Coding. In Visual Communications and Image Processing'88: Third in a Series (Vol. 1001, pp. 424-431). International Society for Optics and Photonics.

Salleh, M. F. M., \& Soraghan, J. (2007). A new multistage lattice vector quantization with adaptive subband thresholding for image compression. EURASIP Journal on Advances in Signal Processing, 2007, 1-11.

Shnain, H. W., Abdullah, M. N., \& Jeiad, H. A. (2020). Implementation of run length encoding using verilog hdL.

Sunitha, P., \& Chitneedi, S. P. (2014). Speech compression for better audibility using wavelet transformation with adaptive kalman filtering. International Journal of Engineering Research and General Science, 2(4), 379-384.

Trabuco, M. H., Costa, M. V., Macchiavello, B., \& Nascimento, F. A. D. O. (2017). S-EMG Signal Compression in One-Dimensional and TwoDimensional Approaches. IEEE journal of biomedical and health informatics, 22(4), 1104-1113.

Wheidima, C. M., Eddie B. L. Filho \& Waldir S. S. J. (2016). Electromyographic Signal Compression Through Image Encoders and Preprocessing Techniques. Journal of communication and information systems, 31(1), 188-197. 